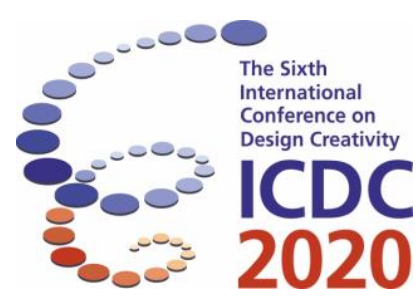

\title{
Too many attributes!: Diminishing the cognitive load of metaphor generation in product design
}

\author{
Alejandra Beghelli ${ }^{1,2}$ and Pablo Prieto ${ }^{3}$ \\ ${ }^{1}$ Faculty of Engineering and Sciences, Universidad Adolfo Ibáñez, Viña del Mar, Chile \\ ${ }^{2}$ Department of Computing, Goldsmiths, University of London, UK \\ ${ }^{3}$ Engineering Design Department, Universidad Técnica Federico Santa María, Chile
}

\begin{abstract}
Many successful products result from a creative ideation process guided by the use of metaphors. Metaphors are used to embed meaning or values in a final product, or as a tool to structure design problems and generate creative solutions. In the former case, the source of inspiration is selected because it naturally exhibits the required traits, whereas, in the latter, the source might be randomly chosen. In both cases, the final result is achieved by transferring one or more attributes from the source to the target product. Given that the number of transferrable attributes is very high compared to the capacity of human's working memory, how can we ensure that we are exploring all possible solutions? Are generated metaphors better when all options are in plain sight? In this paper we describe preliminary results drawn from the use of a tool aimed at facilitating metaphor generation for product concepts.
\end{abstract}

Keywords: metaphors, combinatorial creativity, product design, attributes.

\section{Introduction}

The word "metaphor" originates from the Greek word "metaphora" that means "to transfer". This meaning of the word is key to understand the process by which a metaphor is created: one or more attributes of an abstract or tangible object are transferred to a target product. When doing this transference, both objects merge either in an abstract (like language metaphors) or physical way (like product metaphors). As a result, combinatorial creativity emerges through the unusual combination of familiar ideas (Han et al., 2019).

Previous research on the use of metaphors has focused on main 4 aspects:

- Types of metaphors: in their Structure Mapping Theory (Gentner, 1990), Gentner classifies language metaphors into 3 categories.

○ Attributional metaphors mostly transfer physical aspects from one object to another. The statement "The orange in the sky" (asserting that the sun is like an orange) is an example of a metaphor where the shape and colour of an orange are transferred to the sun. In the design area, this type of metaphor is also known as "surface metaphor" (Casakin, 2004).

- Relational metaphors mostly transfer the relational structure, meaning the relationship between the source's elements or their function. For example, "A roof is like a hat" does not transfer the shape of a hat, but the fact that the hat goes on top of a person and protects them from the elements. In design, this type of metaphor is also 
known as "structural metaphor" (Casakin, 2004). Structural metaphors are perceived as being of better quality than surface metaphors.

- Double metaphors transfer the relational structure and the physical attributes. For example, "Plant stems are drinking straws" refers to the fact that stems not only have a tubular and thin shape (like straws) but also that they have the same function: sucking liquids to nourish a living being.

- Transferred aspects: Hekkert and Cilla (2015) identified eight different properties that can be transferred when applying metaphors to products: form (including shape, outline and colour), sound, material/texture, smell/taste, graphics, interaction, movement/behaviour and name. The first five are physical attributes while the last three might be considered relational attributes.

- Impact of the source's domain: if the target product and the source of inspiration belong to the same or similar domain (e.g. both objects are architectonic products), the metaphor is known as a within-domain metaphor. This type of metaphor is easier to access and tends to be a surface metaphor. However, if target and source belong to different domains, a betweendomain metaphor is generated. These metaphors are more challenging and tend to generate structural metaphors (Casakin, 2004).

- Successful transferences: what specific transferences make a product successful? In (Wang 2016) one hundred products that made it to design competition finals were analysed in terms of the source's domain (artefact, nature materials, animal or plant), the target product's domain (3C products, living goods, furniture, medical products, public facilities), and the transferred aspect (form, colour, texture, interaction, environment, structure).

Note that form, colour and texture aspects refer to physical attributes and thus, their transfer would generate attributional metaphors (Gentner, 1990). In contrast, only structure (relationship between the elements of the object) would produce relational metaphors, according to the classification of (Gentner, 1990). Interaction (how the user interacts with the object, also considered by (Hekkert and Cilla, 2015)) and environment (the context where interaction occurs) aspects are not considered in the classification of (Gentner, 1990).

Based on the analysis of the winning objects, the following rules were conceived:

- If the source of inspiration is an artefact, no matter the target, the transfer of the following pairs might produce a winner product: environment \& structure, or form \& structure. Notice the importance of transferring the structure of the products. This is in line with the observation that structural metaphors generate better results.

$\circ$ If the target product is a living good, no matter the source, then transferring of colour \& environment may produce a winner product. However, if the source is an artefact, it is enough to transfer structure.

The work of (Wang, 2016) can be considered as a 'recipe' where successful transfers depend on several different ingredients (attributes). A 'recipe' has the advantage of decreasing the cognitive load of the person applying it: by sticking to the rules a good idea should be generated. However, it has the drawback of severely limiting the exploration of the solution space. This trade-off led us to the following research question: How can we help designers explore a wider solution space while decreasing the cognitive load of doing so?

This research question has important implications in the education areas of creative design and creative engineering design, while also having implications in computational creativity (a branch of Artificial Intelligence). The development of systematic methods that help deepen our understanding of the creative process is fundamental. On one hand, this speeds up learning and students/novices can quickly internalise this knowledge and use it to enhance their natural creative abilities. They are also pushed to explore wider areas of the solution space. On the other hand, understanding the creative process is key to develop computational tools that aid designers through their creative endeavours, in the medium and long-term.

In this paper we present preliminary results of a card-based method that aims to facilitate attribute transferences between source and target object. The method does not aim to maximise the number of transferred attributes in a single metaphor but to increase the number of metaphors generated by using 
different transfers. The method does not replace the intuitive process followed by designers, it is a supporting tool aimed at facilitating the transfer of attributes that are not easily accessed by human's limited working memory. With this method we expect to deepen the exploration of the solution space. The rest of this paper is composed of four additional sections: Section 2 presents a review of the attributes that can be transferred during metaphor generation. Section 3 introduces the card-based method, while Section 4 presents the results obtained from the card-based method. Section 5 concludes the paper and presents future research lines.

\section{Transferable product attributes}

The results published in (Wang, 2016; Hekkert and Cilla, 2015; Gentner, 1990) show the lack of a unified framework regarding what specific aspects can be transferred when generating a product metaphor.

The two metaphor categories proposed by Gentner, attributional and relational, do not explicitly list sub-categories that might result useful in product design. For example, according to the results in (Wang, 2016), transferring colour might result in a product metaphor with a different level of success than one obtained by transferring form. Nonetheless, for Gentner, these two metaphors belong in the exact same category, even if in terms of product success, they might generate radically different results. This highlights the fact that, when applying metaphors to create products, it is relevant to identify the specific aspect that is being transferred.

The same phenomenon seems to occur for relational metaphors, where very different aspects can be transferred, producing different success rates, yet the aspects are still classified in the same category. For example, the left column of Table 1 shows relational metaphors provided in (Gentner, 1990). On the right column we identify the specific aspect being transferred:

Table 1. Aspects transferred in a relational metaphor

\begin{tabular}{|l|l|}
\hline Relational metaphor & Aspect transferred \\
\hline The moon is a lightbulb & $\begin{array}{l}\text { Function: both objects provide illumination } \\
\text { Behaviour: both are activated at night }\end{array}$ \\
\hline A ladder is like a hill & $\begin{array}{l}\text { Interaction: in both cases the user must make a physical effort to get to the top of } \\
\text { the objects }\end{array}$ \\
\hline $\begin{array}{l}\text { A camera is like a tape } \\
\text { recorder }\end{array}$ & $\begin{array}{l}\text { Function: both objects capture information (visual vs. auditory) } \\
\text { Interaction: in both cases the user must push a button }\end{array}$ \\
\hline A cloud is like a sponge & Function: both hold water that gets released under certain conditions \\
\hline A roof is like a hat & $\begin{array}{l}\text { Function: both objects offer protection } \\
\text { Behaviour: both objects protect from above }\end{array}$ \\
\hline Tree bark is like skin & $\begin{array}{l}\text { Function: both objects offer protection } \\
\text { Behaviour: both objects cover the body and can heal after minor damage }\end{array}$ \\
\hline A tire is like a shoe & $\begin{array}{l}\text { Function: both objects offer protection to a moving part in contact with the ground } \\
\text { Behaviour: both objects wear out with use }\end{array}$ \\
\hline A window is like an eye & $\begin{array}{l}\text { Function: both objects allow the perception of the exterior world } \\
\text { Behaviour: both objects can be opened and closed }\end{array}$ \\
\hline
\end{tabular}

Although all these metaphors are classified as relational, the transferred aspects are different. In this case, we could distinguish function, behaviour, and interaction but there might be others. In a product, the transferred aspect might make a significant difference in terms of market success.

The work of (Hekkert and Cilla, 2015) makes progress by identifying eight aspects that can be transferred: form (including shape, outline and colour), sound, material/texture, smell/taste, graphics, name, interaction and movement/behaviour. However, some physical properties are missing (e.g. temperature, brightness, consistency, size, components/parts, style) as well as important non-physical properties like context of use (space \& time), target users/consumers or accessories.

In Table 2 we summarise the aspects that can be transferred when creating a product through a metaphor. There are two broad categories: physical attributes and usage attributes. The first are all characteristics that can be perceived by human senses. The second category is composed by aspects that define the way the product is used and perceived. This table is the product of six creativity 


\begin{tabular}{|c|c|}
\hline & PHYSICAL ATTRIBUTES \\
\hline & Shape: round, square, rectangle \\
\hline & Colour: any \\
\hline & Graphics: numbers \\
\hline & Mat.: metal, wood, plastic, glass \\
\hline & Texture: soft \\
\hline & Temperature: environment \\
\hline & Smell: none \\
\hline ソ & Taste: none \\
\hline 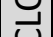 & Sound: tic-toc \\
\hline & Parts: screen, hands, pendulum, \\
\hline & USAGE ATTRIBUTES \\
\hline & Function: record time, wake up \\
\hline & Interaction: look at it, press button \\
\hline & Movement: round (hands), left-right \\
\hline & Accessories: none \\
\hline & Physical Law: electricity or gravity \\
\hline & Context: rooms, wrists \\
\hline a & Emotions: stress, anxiety \\
\hline
\end{tabular}

\begin{tabular}{|l|l|}
\hline & PHYSICAL ATTRIBUTES \\
\hline & Shape: elongated \\
\hline & Colour: silver, gold, metal \\
\hline & Graphics: abstract (handle) \\
\hline Mat.: metal(blade); leather, wood (hilt) \\
\hline & Texture: soft, sharp \\
\hline Temperature: cold \\
\hline & Smell: none \\
\hline \multirow{4}{*}{ Taste: metallic } \\
\hline & Sound: metallic \\
\hline & Parts: blade, guard, hilt, pommel \\
\hline USAGE ATTRIBUTES \\
\hline Function: slash, thrust \\
\hline Interaction: hold it, brandish, sheathe \\
\hline Movements: forward (lunge), parry \\
\hline & Accessories: sheath \\
\hline & Physical Law: sharpness \\
\hline bontext: battle \\
\hline & Emotions: anger, fear \\
\hline
\end{tabular}

Figure 1. Examples of cards describing the attributes of two objects (a and b) and the literal transfer of sword's attributes to a clock (c)

\subsection{Methods and material}

In separate sessions, each group attended a brief introductory session $(15 \mathrm{~min})$ about the use of metaphors as a creative technique. In the same session, they were shown a few examples of product metaphors where different attributes had been transferred, ranging from shape to interaction. Then, they were asked to work in pairs to create new ideas for either a clock (Telematics Engineering) or a lamp (Engineering Design) using metaphors.

We asked both groups to generate ideas for different target objects (clock and lamp) because in first year students are mixed in several modules, and we wanted to avoid students participating first in the experiment to share ideas with the students participating later on. All students were instructed to use the cards to facilitate the idea generation process.

Participants were left free to generate as many ideas as they wanted and to select any card to be used as a source. For each generated idea, the students had to identify the target object (either a clock or a lamp), the source (any of the objects/concepts described by the cards), and the attribute(s) being transferred.

The group working on ideas for a clock received the cards: car race, curtains, forest, guillotine, heart, maze, monkey, playing card, shoe, sword, windmill, and window. The concept 'clock' belongs to the conceptual domains of 'time' and 'measurement'. None of the sources belong to either of these conceptual domains. Thus, the generated metaphors will necessarily be between-domain ones. That is, metaphors that will impose a higher cognitive load in participants (Ozkan, 2013).

The group working on ideas for a lamp received the remaining cards: butterfly, door, firefly, floor, giraffe, jellyfish, Picasso, sun, tree, virus, and washbasin. The concept 'lamp' belongs to the conceptual domains of 'lightning' and 'furniture'. The concepts 'sun' and 'firefly' belong to the 'lightning' domain. None of the sources belong to the 'furniture' domain. Thus, if the proposed tool helped decrease the cognitive load, between-domain metaphors will also be generated despite the fact that sources from the same domain can be selected.

\subsection{Results}

A total of seventy-five ideas were generated for a clock (average number of ideas per group: 2.6) and one-hundred-and-six for a lamp (average number of ideas per group: 3.4). Two aspects might explain the difference in the number of generated ideas. First, the different backgrounds of both groups: students generating ideas for a clock were from a Computer Science, Electronics, \& Networks focused programme (not usually pursued by creative people), whereas students generating ideas for a lamp were from a programme with a focus on design. Secondly, all sources for a clock are from a different conceptual domain, and therefore more complex. 


\subsubsection{Cognitive load and anomalies}

A first important finding is that no 'anomalies' were generated. An 'anomaly' is a design solution that does not use any feature from the source at all. According to the findings reported in (Ozkan, 2013), a $26 \%$ of the solutions generated by first-year students working on a metaphor generation task were 'anomalies'. In this study, such situation did not occur. This suggests that the cognitive challenge of searching for attributes to transfer was eased by the use of the cards, given that all participants generated at least one idea for which they found at least one attribute to transfer.

\subsubsection{Cognitive load and conceptual domains}

We analysed the solutions in terms of the selected sources for the metaphor. We expected that students generating metaphors for a lamp would make higher use of in-domain metaphors ('sun' and 'firefly'). Figure 2 shows the number of ideas generated for each source selected as inspiration for a clock (left) and a lamp (right).
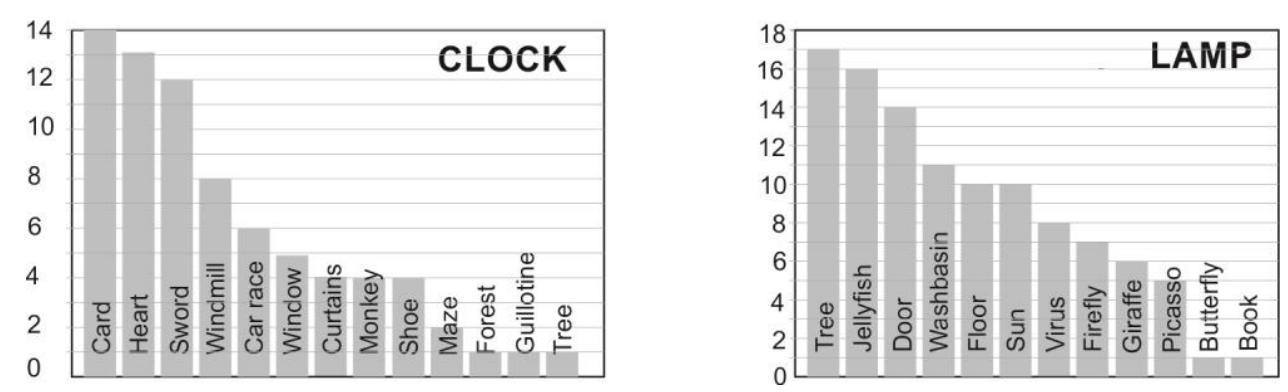

Figure 2. Number of ideas generated per source for a clock (left) and a lamp (right)

It can be seen that the sources being selected the most (about $50 \%$ of ideas were generated using them as a source of inspiration) do not belong to the same conceptual domain as the target. These results are encouraging: previous research has shown that between-domain metaphors impose a higher cognitive load than in-domain metaphors (Ozkan, 2013). The fact that most metaphors generated were from the between-domain category, suggests that having all attributes in plain sight contributes to decrease the cognitive load.

\subsubsection{Cognitive load and metaphor quality}

Next, we studied the quality of the metaphors generated. In theory, between-domain metaphors tend to be structural -and thus are deeper and better- metaphors. Structural metaphors (also called deep metaphors) are the ones resulting from the transference of a non-salient quality (Cila, 2014). Since non-salient qualities are not the ones at the top of student's minds, this method would help explore a wider solution space and, eventually, consistently lead to deeper metaphors.

To verify whether deeper metaphors are generated, we analysed the attributes transferred during the creative process. Figures 3 and 4 show the percentage of the ideas generated for each attribute. Figure 3 was built only considering ideas for a clock that came from the most commonly used sources in this experiment: 'playing card', 'heart', and 'sword'. Analogously, Figure 4 was built considering the lamp ideas generated using the sources 'tree', 'jellyfish', and 'door'.

It can be observed that the transference of physical attributes, leading to the generation of surface metaphors, is the most common choice. In fact, shape was the most transferred attribute. This is in line with previous research stating that surface metaphors are easier to access or identify. Contrary to our expectation, interaction attributes were seldom selected. However, when selected they were likely to generate structural metaphors. For example, in the case of a clock inspired by a heart, one metaphor transferred the function of the heart: make blood to travel through the circulatory system. The idea of the clock was a wristwatch with a simple circular line. Every minute, the line is filled with a red colour following the clockwise direction, thus, imitating the function of the heart: pushing time through the line. In the case of a lamp inspired by a tree, the lamp had the shape of a tree where leaves would imitate the photosynthesis process using solar panels to collect energy and power the lamp. 

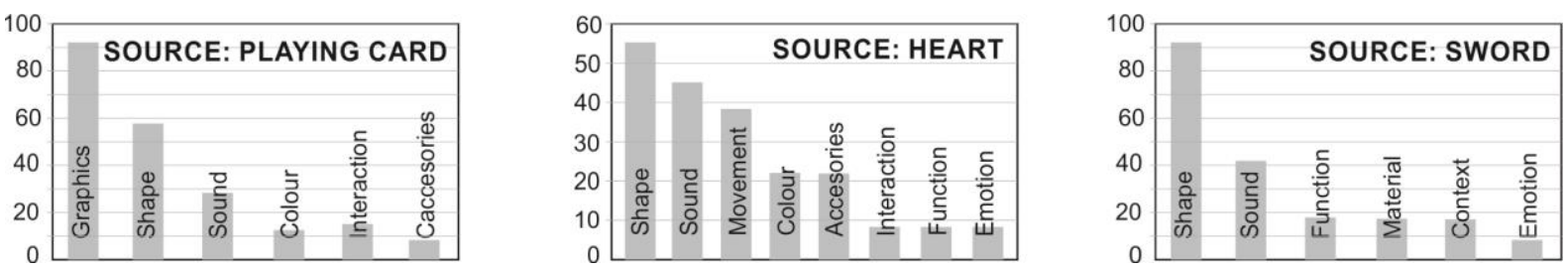

Figure 3. Attributes being transferred to a clock from the 3 most used sources of inspiration
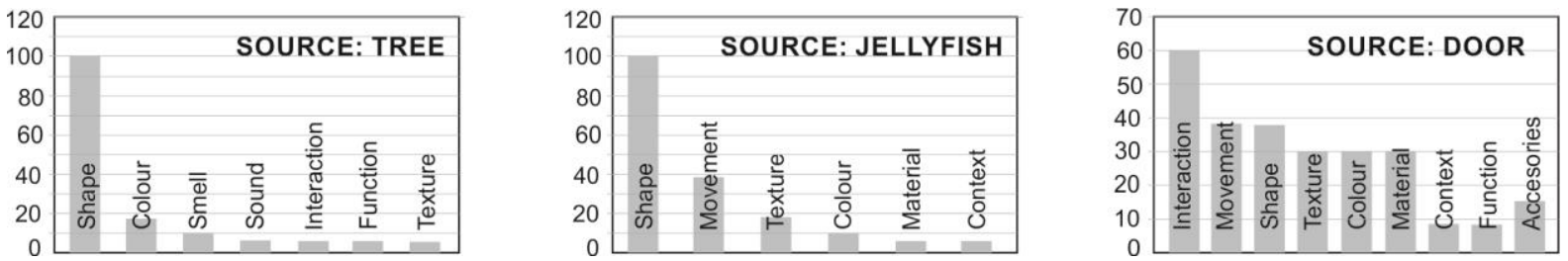

Figure 4. Attributes being transferred to a lamp from the 3 most used sources of inspiration

One interesting observation is that participants did not necessarily select the most salient feature to generate a metaphor. For example:

- In the case of 'sword', previous research on salient attributes spontaneously mentioned by people showed that shape and function account for $48 \%$ of mentions (McRae et al., 2005). The attribute 'sound' is not one of the most salient aspects of a 'sword'. However, it was the second most transferred attribute in our experiment, accounting for the $40 \%$ of the metaphors generated when using 'sword' as a source.

- In the case of 'door', salient attributes spontaneously mentioned by people are the material (wood/metal), the fact that is has a lock, and -in third place- the fact that it can be opened or closed (McRae et al., 2005). In our experiment instead, the attributes of interaction and movement (open and closing the door) dominated the transfers, whilst material and accessories were not the most used attributes.

Both examples highlight the fact that the cards do help retrieve non-salient attributes that otherwise would not be used due to the cognitive difficulty associated with accessing them. Further research should verify whether the same phenomenon occurs with other sources not included in the study of McRae et al. (2005).

Finally, it is worth mentioning that -despite having many attributes to select from in plain sight- most participants preferred a reduced set of attributes to work with. This is an area for further improvement.

\section{Conclusions and Future Work}

In this paper we presented a method to support the generation of design concepts by using metaphors. The method aimed to reduce the cognitive load designers face when deciding what attributes to transfer. On the positive side, results suggest that the cards helped:

- Avoid 'anomalies', thus decreasing the cognitive load of searching for attributes to transfer

- Produce between-domain metaphors, cognitively more demanding than in-domain metaphors

- Select non-salient attributes from the source, which are usually difficult to access.

On the negative side, results suggest that the cards did not encourage the selection of interaction attributes, as originally expected. To improve this, an approach that forces such selections might be used. For example, by having to generate a metaphor using a randomly selected attribute. A card like the one shown in Figure 5 -where a spinning wheel selects a random source and attribute- might prove to be more useful for this. Testing this card (in an analogue and digital version) with novice and experienced designers is part of further research. 


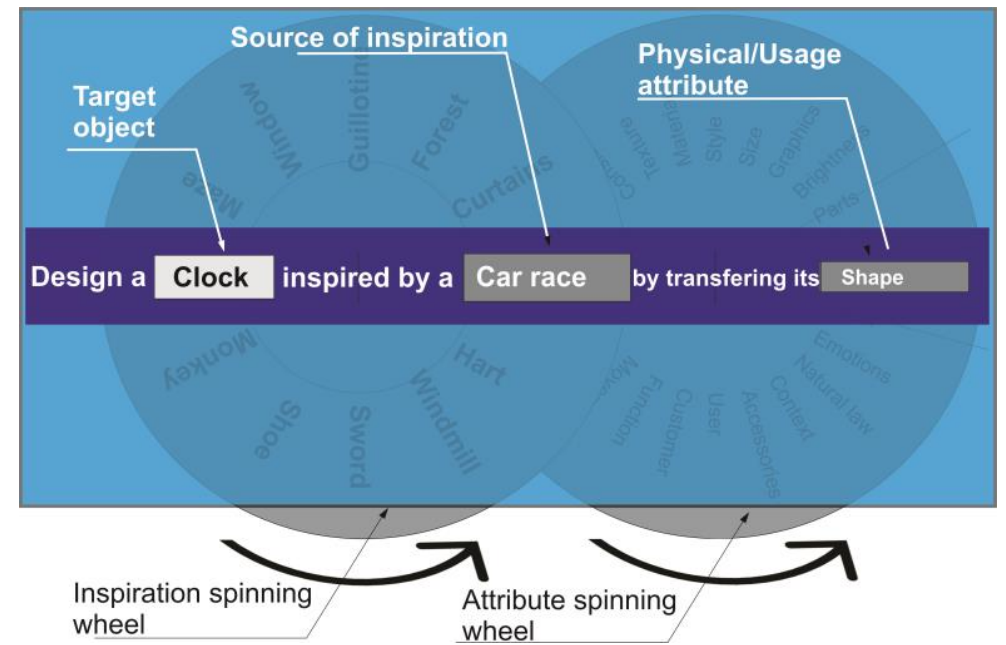

Figure 5. Card to support the generation of metaphors by random selection of source and attribute to transfer

\section{Acknowledgements}

Financial support from Universidad Adolfo Ibáñez, Universidad Técnica Federico Santa María and Basal Project FB-008 is gratefully acknowledged.

\section{References}

Casakin, H. (2004) Visual Analogy as a Cognitive Strategy in the Design Process: Expert Versus Novice Performance. Journal of Design Research, 4(2), 197-217

Chandler, P. and Sweller, J. (1991), Cognitive Load Theory and the Format of Instruction, Cognition and Instruction, Vol.8 No. 4, pp. 293-332. https://doi.org/10.1207/s1532690xci0804_2

Cila, N., Hekkert, P. And Visch, V. (2014) Digging for Meaning: The Effect of a Designer's Expertise and Intention on Depth of Product Metaphors, Metaphor and Symbol, 29(4), 257-277

McRae, K., Cree, G., Seidenberg, M. and McNorgan, C. (2005) Semantic feature production norms for a large set of living and non living things. Behaviour Research Methods, Instruments and Computers, 37 (4), 547-559

Miller, G. A. (1956). The magical number seven, plus or minus two: Some limits on our capacity for processing information. Psychological Review, 63 (2): 81-97.

Wang, H. (2016). Winning formulas for metaphor design: A case study of design competitions, In Proceedings of DESIGN 2016, Dubrovnik, Croatia.

Han, J., Park, D. Shi, F., Hua, M. and Childs, P. (2019). Three driven approaches to combinational creativity: Problem-, similarity- and inspiration-driven. Proceedings of the Institution of Mechanical Engineers, Part C: Journal of Mechanical Engineering Science, 233(2), 373-384

Ozkan, O. and Dogan, F. (2013). Cognitive strategies of analogical reasoning in design: Differences between expert and novice designers. Design Studies 34, 161-192 